Research Article Plant Genetics

\title{
Intense proliferation of rDNA sites and heterochromatic bands in two distantly related Cuscuta species (Convolvulaceae) with very large genomes and symmetric karyotypes
}

\author{
Amália Ibiapino $^{1}$, Miguel Ángel García ${ }^{* 2,3}$, Mihai Costea ${ }^{4}$, Saša Stefanović ${ }^{2}$ and Marcelo Guerra ${ }^{1} \mathbb{D}$ \\ ${ }^{1}$ Universidade Federal de Pernambuco, Departamento de Botânica, Recife, PE, Brazil. \\ ${ }^{2}$ University of Toronto Mississauga, Department of Biology, Mississauga, On, Canada. \\ ${ }^{3 *}$ Royal Botanic Gardens Kew, Richmond, Surrey, United Kingdom. \\ ${ }^{4}$ Wilfrid Laurier University, Department of Biology, Waterloo, On, Canada.
}

\begin{abstract}
The genome size varies widely among angiosperms but only a few clades present huge variation at a low phylogenetic level. Among diploid species of the genus Cuscuta the genome size increased enormously in at least two independent lineages: in species of subgenus Monogynella and in at least one species $(C$. indecora) of the subgenus Grammica. Curiously, the independent events lead to similar karyotypes, with $2 n=30$ mostly metacentric chromosomes. In this paper we compared the patterns of heterochromatic bands and rDNA sites of $C$. indecora and $C$. monogyna, aiming to evaluate the role of these repetitive fractions in these karyotypes. We found out that the large genomes of these species were incremented by a huge number of small heterochromatic $\mathrm{CMA}^{+}$and $\mathrm{DAPl}^{+}$bands and $5 \mathrm{~S}$ and $35 \mathrm{rDNA}$ sites, most of them clearly colocalized with $\mathrm{CMA}^{+}$bands. Silver nitrate impregnation revealed that the maximum number of nucleoli per nucleus was low in both species, suggesting that some of these sites may be inactive. Noteworthy, the tandem repeats did not generate large bands or sites but rather dozens of small blocks dispersed throughout the chromosomes, apparently contributing to conserve the original karyotype symmetry.
\end{abstract}

Keywords: CMA and DAPI staining, dodders, genome size, karyotype symmetry, rDNA sites.

Received: February 28, 2019; Accepted: April 6, 2020.

\section{Introduction}

The genus Cuscuta L. (Convolvulaceae), commonly known as dodders, consists of approximately 200 species (Yuncker, 1932; Costea et al., 2015) of hemiparasitic or holoparasitic herbs, and is nearly cosmopolitan in distribution. Taxonomically, this group is difficult due to interspecific hybridizations, infraspecific variability, and strong vegetative reduction associated with diminished or complete lack of photosynthetic activity resulting in morphological parallelism among species (Stefanović and Costea, 2008; Costea et al., 2015). Cytologically, however, Cuscuta is one of the plant genera with the largest variability in genome size $(1 \mathrm{C}=0.48 \mathrm{pg}$ to $1 \mathrm{C}=32.77 \mathrm{pg})$ and perhaps the only plant genus with species having both monocentric and holokinetic chromosomes (Pazy and Plitmann, 1995; McNeal et al., 2007; Leitch et al., 2010; Guerra et al., 2019).

Cuscuta is currently divided into four subgenera: $\mathrm{Mo}$ nogynella, represented by approximately 15 species of the

Send correspondence to: Marcelo Guerra, Universidade Federal de Pernambuco, Departamento de Botânica, Avenida Professor Moraes Rego, Cidade Universitária, CEP 50.372-970, Recife, PE, Brazil. E-mail: msfguerra@ hotmail.com.

${ }^{*}$ Current address: Real Jardin Botánico, CSIC, Madrid, Spain.
Old World; Grammica, comprising about 150 species mostly from the Americas; Cuscuta, with 20-25 species originally from the Old World; and Pachystigma, a small group of five species endemic to South Africa (Costea et al., 2015). The scarce chromosome counts available for only 35 species of this genus indicate that most diploid species present $2 n=28$ or $2 n=30$, and that each subgenus followed a distinct karyotype trend. There are very large chromosomes in Monogynella (Pazy and Plitmann, 1995), holokinetic chromosomes in the subgenus Cuscuta (García and Castroviejo, 2003), large DNA content variation (2C = 0.96 to $65.54 \mathrm{pg}$ ) in Grammica (McNeal et al., 2007; Kubesová et. al., 2010), and strongly bimodal karyotypes in Pachystigma (García et al., 2019). Phylogenetically, the genus Cuscuta is clearly nested within Convolvulaceae (Stefanović et al., 2002; Stefanović and Olmstead, 2004), a family otherwise characterized by small genome size $(2 \mathrm{C} \leq$ $4.5 \mathrm{pg}$ ) (Kew Plant DNA C-values Database; http://data.kew.org/cvalues/), with predominantly symmetrical karyotypes, small chromosomes, and chromosome number $2 \mathrm{n}=30$ in most genera and species (e.g., Yen et al., 1992; Pitrez et al., 2008). 
Extreme increase in genome size may have occurred at least twice during the evolution of the genus Cuscuta: across species of the subgenus Monogynella (C. exaltata Engelm., 2C $=41.86$ pg; $C$. lupuliformis Krock., 2C = $44.93 \mathrm{pg}$ ), and in at least one species of the subgenus Grammica [C. indecora Choisy, with 2C $=65.54 \mathrm{pg}$ (McNeal et al., 2007)], placed in the small section Indecorae. Likewise, the largest chromosome size for the genus was reported for two other Monogynella species, $C$. reflexa Roxb. (Kaul and Bahn, 1974) and C. monogyna Vahl. (Pazy and Plitmann, 1995; García and Castroviejo, 2003), and for C. indecora (Fogelberg, 1938; García et al., 2019). Figure 1 illustrates the phylogenetic relationships among Cuscuta subgenera and the relative position of $C$. indecora and C. monogyna (based on García et al., 2014; Stefanović et al., 2007; Costea et al., 2015). Note that $C$. indecora is not monophyletic as currently circumscribed.

In spite of the huge variation in chromosome size across the genus, all Monogynella and Grammica species display symmetrical or nearly symmetrical karyotypes (García et al., 2019), suggesting that the evolution of the biggest genomes occurred without structural rearrangements that could change the chromosome morphology. Large genome expansions, as observed in Cuscuta, are more commonly due to a burst of one or a few retroelements and/or satellite DNA sequences (Michael, 2014; Garrido-Ramos, 2017). Because

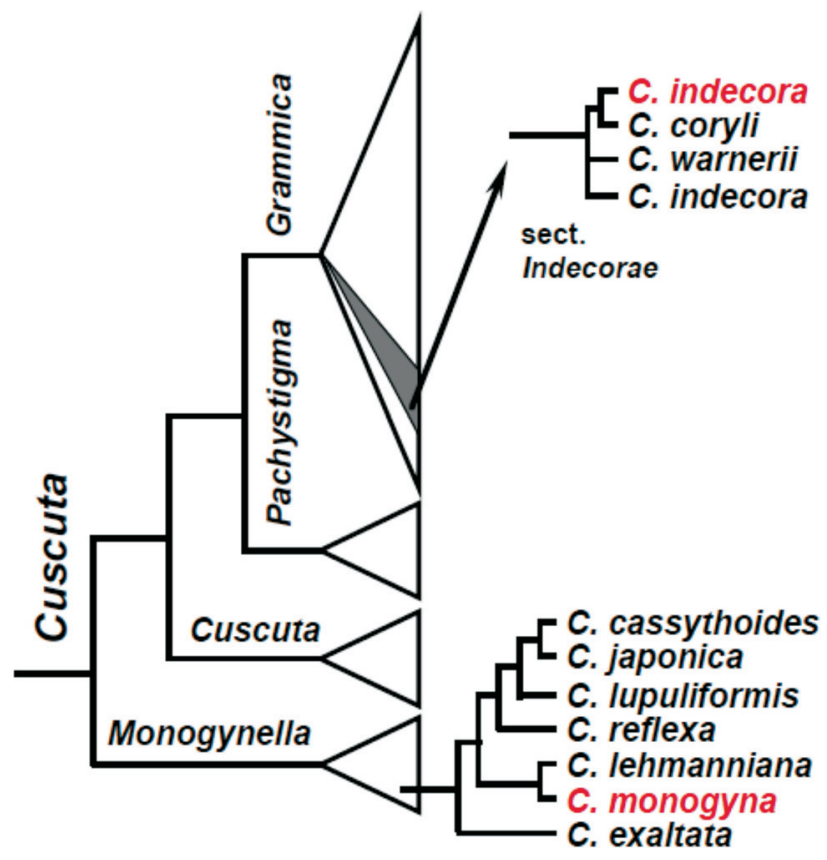

Figure 1 - Schematic overview of the phylogenetic relationships in $\mathrm{Cus}$ cuta derived from sequence data from plastid ( $\operatorname{rn} L-F, r b c L)$ and nuclear (nrITS, nrLSU) sources and analyzed with a range of analytical methods (modified from Costea et al., 2015). Infrageneric classification is provided above branches on the left, and more detailed relationships among species of Cuscuta sect. Indecorae and Cuscuta subgenus Monogynella are shown on the right (Stefanović et al., 2007; García et al., 2014). Species of particular interest for this study are highlighted in red. Note that C. indecora is not monophyletic as currently circumscribed. satellite DNA families are organized in blocks of tandemly repeated sequences, they are usually not included in the genome sequencing and hence its real role in the genome size variation is poorly known. Recent analyses of satellite DNA families detected by next-generation sequencing (NGS) combined with appropriate bioinformatic tools and chromosome in situ hybridization (FISH) revealed that most satellites colocalized with the classical chromosome bands (Ruiz-Ruano et al., 2016; Palacios-Gimenez et al., 2017; Robledillo et al., 2018).

Chromosome banding using base-specific fluorochromes, mainly the DNA ligand chromomycin A3 (CMA) and 4',6-diamidino-2-phenylindole (DAPI), which bind preferentially to AT-rich and GC-rich sequences respectively, reveal most of the heterochromatin content of the karyotype (Barros e Silva and Guerra, 2010). The only Cuscuta species investigated by chromosome banding and FISH using 5S and 35S rDNA probes were C. approximata Bab. of subgenus Cuscuta (Guerra and García, 2004), and the three species of subgenus Grammica section Denticulatae (Ibiapino et al., 2019), which exhibited a variable number of CMA and DAPI bands and rDNA sites.

We undertook the current work to estimate the genome size of C. monogyna and C. indecora and to provide a detailed karyotype analysis of both species, including the heterochromatic bands and rDNA sites, with an ultimate aim to evaluate the role of the repetitive fractions in these convergent genome expansions and maintenance of karyotype symmetry in these independent lineages.

\section{Material and Methods}

\section{Plant material}

One sample of Cuscuta monogyna and four samples of $C$. indecora were analyzed. The samples investigated with their collection information, voucher number, herbaria where the vouchers are deposited and karyotype data are presented in Table 1. Seeds of both species were scarified with concentrated sulfuric acid for 60-90 s, rinsed several times with distilled water, and germinated on wet filter paper in Petri dishes. Seedlings were cultivated in the greenhouse of the University of Toronto Mississauga, using coleus [Plectranthus scutellarioides (L.) R. Br.] as a host. Seedlings of C. monogyna were also cultivated in the Federal University of Pernambuco (Recife, Brazil), where most cytological analyses and genome size estimation were conducted. Vouchers are deposited in the herbarium of the University of Toronto Mississauga (TRTE).

\section{Slide preparation and chromosome staining}

For mitotic analyses, shoot tips were pretreated in $0.2 \%$ colchicine for 24 hours at $10{ }^{\circ} \mathrm{C}$, fixed in a 3:1 ethanol-acetic acid solution, and subsequently stored at $-20{ }^{\circ} \mathrm{C}$. For meiotic analyses, young flower buds were directly fixed and stored as above. For cytological analyses, we fol- 
Table 1 - Samples of Cuscuta monogyna and C. indecora investigated, with respective voucher, collection locality, chromosome number observed in meiosis (n) or mitosis (2n), and genome size (2C).

\begin{tabular}{|c|c|c|c|c|c|}
\hline Species & Voucher & Locality & $n$ & $2 n$ & $2 \mathrm{C} \pm \mathrm{CV}$ \\
\hline $\begin{array}{l}\text { Cuscuta monogyna } \\
\text { Vahl }\end{array}$ & UTM-1348 & Israel: Kursi; dat: 2012 & 15 & 30 & $66.08 \pm 0.27$ \\
\hline \multirow[t]{4}{*}{ C. indecora Choisy } & UTM-1568 & $\begin{array}{l}\text { Supplied by SAGARPA (Secretaría de Agricultura, Ganade- } \\
\text { ria, Desarrollo Rural, Pesca y Alimentación), México }\end{array}$ & 15 & 30 & 45.582 .66 \\
\hline & $\begin{array}{l}\text { Stefanović SS-16-74, } \\
\text { TRTE }\end{array}$ & $\begin{array}{l}\text { USA: New Mexico; Chaves Co., Roswell, corner E McCune and } \\
\text { S Main St (Hwy 285); dat: } 16 \text { Aug } 2016\end{array}$ & 15 & & \\
\hline & $\begin{array}{l}\text { Stefanović SS-16-53, } \\
\text { TRTE }\end{array}$ & $\begin{array}{l}\text { USA: New Mexico; Socorro Co., on Pueblitos Rd., 1/3 mi E of } \\
\text { Escondida Bridge Park ( } 1 / 2 \mathrm{mi} \text { E of Hwy 408); dat: } 9 \text { Aug } 2016\end{array}$ & 15 & 30 & \\
\hline & $\begin{array}{l}\text { Stefanović SS-16-77 b, } \\
\text { TRTE }\end{array}$ & $\begin{array}{l}\text { USA: New Mexico; Chaves Co., Bottomless Lakes Rd., } 3 \text { mi S of } \\
\text { Hwy 380. } 17 \text { August 2016. } 3321^{\prime} 31^{\prime \prime N ~} 10420 ' 16 " \mathrm{~W}\end{array}$ & & & $50.03 \pm 0.05$ \\
\hline
\end{tabular}

lowed the same protocols used for other Cuscuta species (Guerra and García, 2004). The fixed material was washed in distilled water, digested in a $2 \%(\mathrm{w} / \mathrm{v})$ cellulase (Onozuka) $/ 20 \%$ (v/v) pectinase (Sigma) solution at $37^{\circ} \mathrm{C}$ for 60 min, squashed in a drop of $45 \%$ acetic acid and the coverslip removed in liquid nitrogen.

For CMA/DAPI staining, the slides were aged for three days, and stained for $60 \mathrm{~min}$ with CMA $(0.1 \mathrm{mg} / \mathrm{mL})$ and $30 \mathrm{~min}$ with DAPI $(1 \mu \mathrm{g} / \mathrm{mL})$. The slides were then maintained in the dark for three days before analysis under an epifluorescence Leica DMLB microscope. The images were captured with a Cohu CCD video camera using Leica QFISH software and were later optimized for better contrast and brightness using Adobe Photoshop CS3 version 10.0 .

In situ hybridization was performed according to Pedrosa et al. (2002), with small modifications. A 500 bp 5S rDNA clone (D2) of Lotus japonicus (Regel) K. Larsen, labelled with Cy3-dUTP (Amersham), and a $6.5 \mathrm{~kb} 35 \mathrm{~S}$ rDNA clone (R2) of Arabidopsis thaliana (L.) Heynh., labelled with digoxigenin-11-dUTP, were used as probes. The labelling was done by nick translation. The $35 \mathrm{~S}$ rDNA probe was detected with sheep anti-digoxigenin FITC (Roche) and amplified with rabbit anti-sheep FITC (Dako). The hybridization mix contained formamide $50 \%(\mathrm{v} / \mathrm{v})$, dextran sulphate $10 \%(\mathrm{w} / \mathrm{v}), 2 \mathrm{SSC}$ and $5 \mathrm{ng} / \mu \mathrm{L}$ of each probe. Both chromosomes and probes were denatured at 75 ${ }^{\circ} \mathrm{C}$ for $10 \mathrm{~min}$ and hybridized at $37{ }^{\circ} \mathrm{C}$ for $18 \mathrm{~h}$. The post-hybridization washes were performed in $0.1 \mathrm{SSC}$ at 42 ${ }^{\circ} \mathrm{C}$ for $15 \mathrm{~min}$, the slides were counterstained with DAPI 2 $\mu \mathrm{g} / \mathrm{mL}$ and mounted in Vectashield H-1000 (Vector). The cells previously acquired with CMA/DAPI staining were photographed again and the images were optimized as before.

Because both species presented a high number of rDNA sites, we analyzed the number of nucleoli per nucleus by silver nitrate impregnation to check if there was a real increment in the number of active nucleolus organizer regions (NORs). In this case, a drop of 50\% silver nitrate diluted in distilled water was added to slides containing a large number of interphase nuclei from young shoot tips, covered with a coverslip, and maintained at $60{ }^{\circ} \mathrm{C}$ in water bath for 1-2 hours [slightly modified from Kodama et al. (1980)]. When nucleoli were clearly differentiated, the slides were washed, air dried, and mounted in glycerol.

\section{Chromosome length measurement and flow cytometry}

Chromosome size estimation was based on measurements of the four best metaphases of each species, using Adobe Photoshop CS3 software version 10.0. Chromosome arm ratio (length of the long arm/length of the short arm) was used to classify chromosomes as metacentric (1.00-1.49) or submetacentric (1.50-2.99), according to Guerra (1986). For flow cytometry, a suspension of nuclei from shoot tips was prepared using WPB buffer (Loureiro et al., 2007). The cells were stained with propidium iodide and the nuclear DNA amount was estimated using a CyFlow SL flow cytometer (Partec, Görlitz, Germany). As an internal control young leaves of Vicia faba L. ssp. faba 'Inovec' $(2 \mathrm{C}=26.9 \mathrm{pg}$; Doleel et al., 1991) were used. The final $2 \mathrm{C}$ value was based on three different measurements for each sample using the equation "Sample peak mean/Standard peak mean 2C DNA content of internal control (pg)" and the software FloMax (Partec) for data processing.

\section{Results}

\section{Chromosome number, size, morphology and DNA amount}

The two species displayed $2 \mathrm{n}=30$ large chromosomes with similar symmetrical karyotypes (Figures. 2 and 3). Secondary constrictions were observed on a single pair of metacentric chromosomes in both species, although they were not always visible. They were located interstitially in C. monogyna (Figure 2d) and proximally in C. indecora (upper insets in Figure 3b). In meiosis, both species presented regular chromosome pairing with 15 bivalents. In $C$. monogyna, there were 13 metacentric pairs varying from 14.49 to $21.60 \mu \mathrm{m}$ (arm ratio: 1.05 to 1.29 ) and two submetacentrics displaying 12.41 and $13.65 \mu \mathrm{m}$ in length (arm ra- 


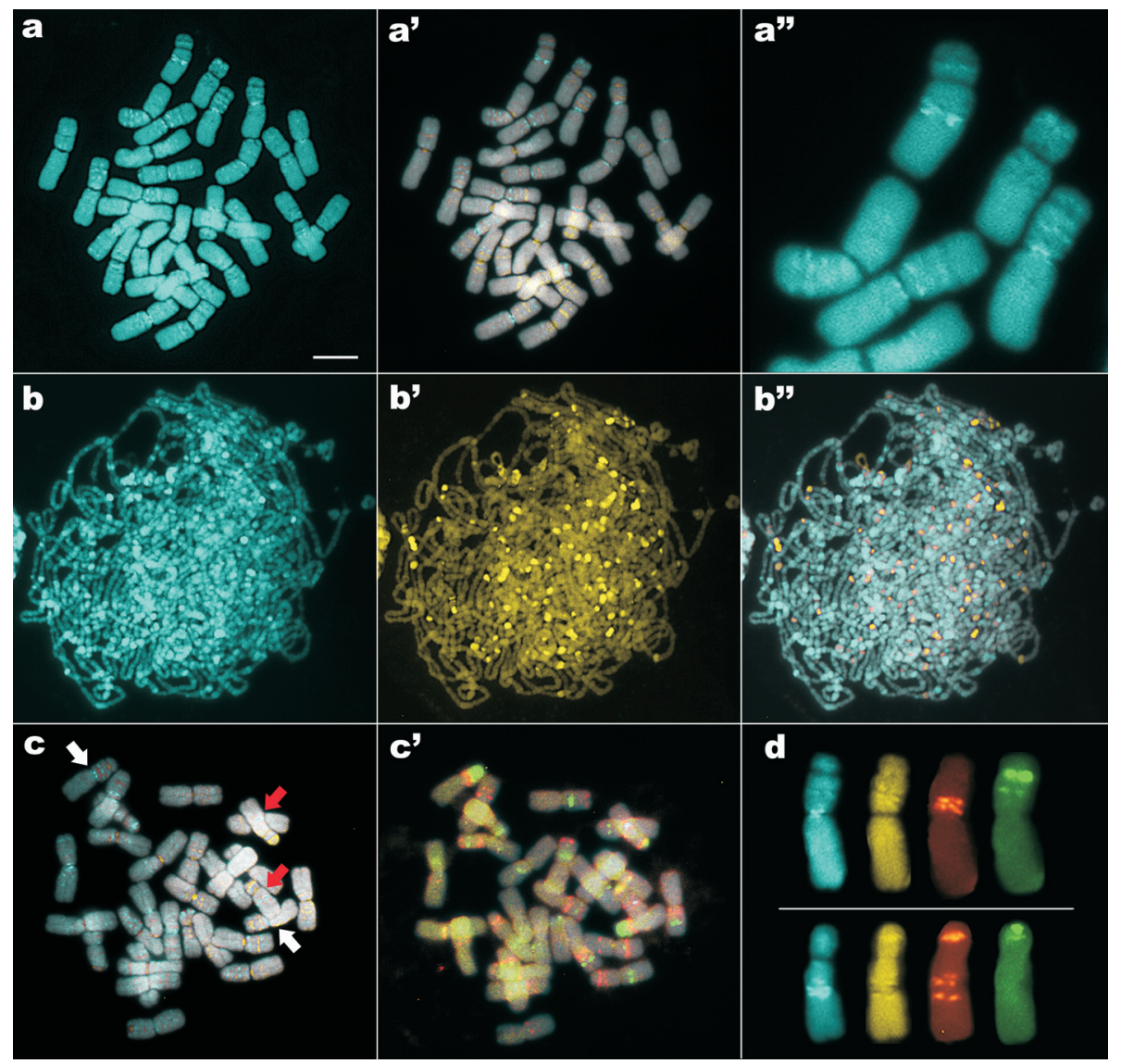

Figure 2 - CMA/DAPI bands and rDNA sites in Cuscuta monogyna. (a-a") Metaphase showing DAPI bands (a), DAPI and CMA merged images (a) and enlarged images of some chromosomes with several DAPI bands (a"). (b-b") Zygotene stained with DAPI (b), CMA (b') and merged images (b"). (c, c') Metaphase showing CMA and DAPI bands (c) and rDNA sites (c'). White and red arrows indicate the chromosome pairs bearing, respectively, the first and the second largest pairs of 35S rDNA sites. (d) First (upper row) and second (lower row) largest pairs of satellited chromosomes from another metaphase showing heterochromatic bands and rDNA sites. Observe that the centromere in the metacentric pair was $\mathrm{DAPI}^{+} / \mathrm{CMA}^{-}$whereas in the other pair it was negative for DAPI and undifferentiated for CMA. Blue $=$ DAPI; yellow $=$ CMA; orange $=5 \mathrm{~S}$ rDNA; green $=35 \mathrm{~S}$ rDNA. Bar in (a) corresponds to $10 \mu \mathrm{m}$ (not valid for a" and d).

tio: 1.91 and 2.59). Cuscuta indecora (UTM-1568) had 14 metacentric pairs varying in size from 13.66 to $18.25 \mu \mathrm{m}$ (arm ratio:1.00 to 1.31) and one submetacentric with an average size of $10.77 \mu \mathrm{m}$ (arm ration: 2.50). The genome size was higher in $C$. monogyna $(2 \mathrm{C}=67.58 \pm 0.27 \mathrm{pg})$ than in C. indecora. The two samples of $C$. indecora analyzed by flow cytometry presented different results: $2 \mathrm{C}=50.03 \pm$ $0.05 \mathrm{pg}$ (SS-16-77b) and 2C $=45.58 \pm 2.66 \mathrm{pg}$ (UTM1568). The former estimation was obtained from shoot tips of young plantlets whereas the latter one was from an adult plant growing in greenhouse. However, this variation may also be due to differences between populations as indicate in Figure 1.

\section{CMA/DAPI bands and rDNA sites}

Cuscuta monogyna showed a very large number of small $\mathrm{CMA}^{+} / \mathrm{DAPI}^{-}$and $\mathrm{CMA}^{-} / \mathrm{DAPI}^{+}$bands, resulting in a stripped appearance of some chromosome arms (Figure 2a-a"). Most of the bands were weakly contrasted, especially the $\mathrm{CMA}^{+}$ones, and the vast majority were located on interstitial positions, although there were also some terminal, proximal, and a few centromeric bands. The whole heterochromatin of C. monogyna was more clearly seen in early-pachytene nuclei (Figure 2b-b"), allowing to count almost $90 \mathrm{CMA}^{+} / \mathrm{DAPI}^{-}$bands and near $80 \mathrm{DAPI}^{+} / \mathrm{CMA}^{-}$ bands. The exact number of bands and rDNA sites was difficult to ascertain because some of them were too closely positioned, too small, or weakly labelled.

In situ hybridization revealed nearly 36 sites of $5 \mathrm{~S}$ rDNA and 30 sites of $35 \mathrm{~S}$ rDNA in C. monogyna (Figure $\left.2 c^{\prime}\right)$. Most sites were interstitials, except three pairs of $5 \mathrm{~S}$ and one pair of $35 \mathrm{~S}$ rDNA, which were terminally located. Noteworthy, only six pairs of $35 \mathrm{~S}$ rDNA sites and near half of the 5S rDNA sites of C. monogyna were clearly colocalized with $\mathrm{CMA}^{+}$bands (Figure 2c, c'). Although several rDNA sites were located very close to DAPI ${ }^{+}$bands, detailed analysis revealed that none of them were colocalized with $\mathrm{DAPI}^{+}$bands.

Each chromosome pair of C. monogyna had at least one or more heterochromatic bands and rDNA sites, allow- 


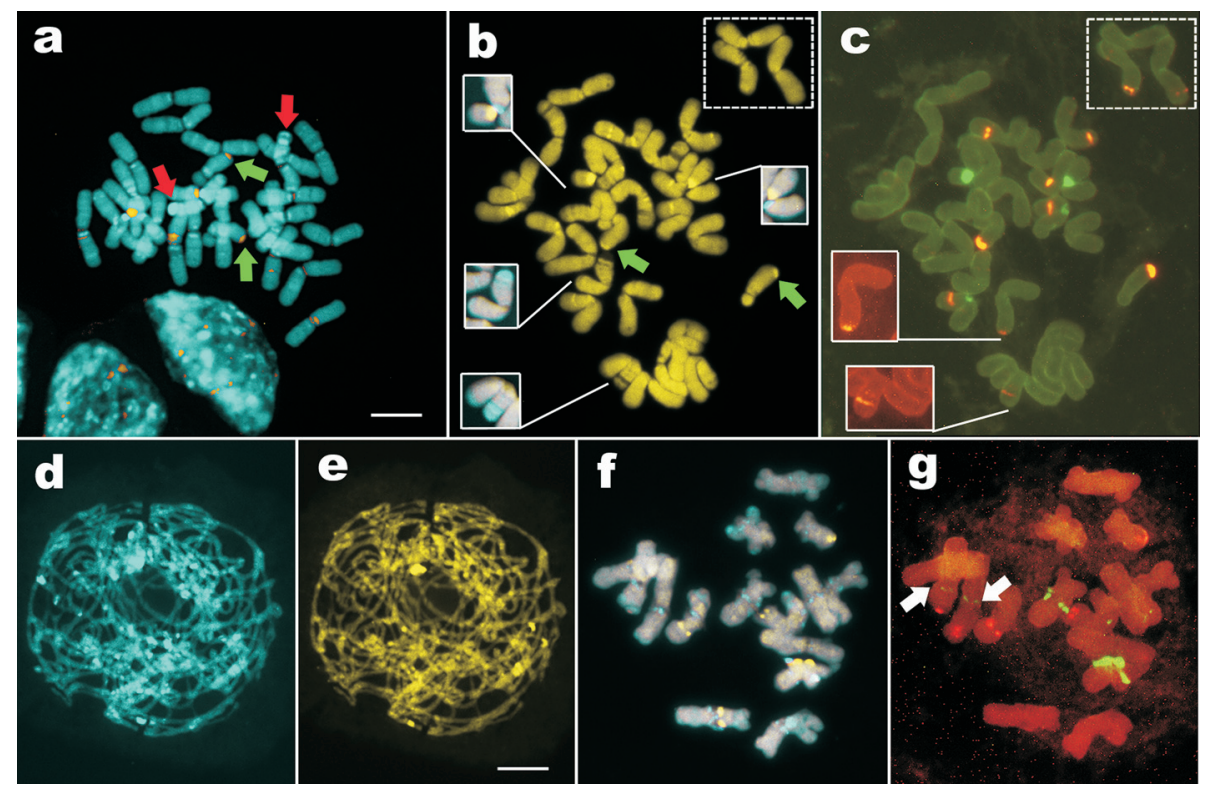

Figure 3 - CMA/DAPI bands and rDNA sites in Cuscuta indecora (a) Metaphase showing merged DAPI and CMA images (red arrows = terminal DAPI bands; green arrows = terminal CMA bands). (b, c) Metaphase with CMA bands (b) and 5S (orange) and 35S (green) rDNA sites (c). Three chromosomes were outside the picture (dashed square). Insets in (b) show chromosome pair with proximal secondary constriction (up) and terminal DAPI bands (down) and in (c) show weak sites. Green arrows in (a) and (b) point to CMA ${ }^{+}$bands co-localized with 5S rDNA sites. (d, e) Zygotene stained with DAPI (d) and CMA (e). (f, g) Diakinesis showing main bands (f) and rDNA sites (g) with two weak 35S rDNA sites (white arrows). Bar in (a) corresponds to $10 \mu \mathrm{m}$.

ing for an easy identification of every chromosome pair. The two pairs bearing the largest 35S rDNA sites of the complement illustrated very well the use of these markers for chromosome identification. The largest $35 \mathrm{~S}$ rDNA site was located on the shorter arm of a metacentric pair, colocalized with a weak $\mathrm{CMA}^{+}$band negatively stained by DAPI (white arrows in Figure 2c and selected chromosomes from another metaphase in Figure 2d upper row). This chromosome arm also had a smaller 35S rDNA site and two 5S rDNA sites. The second largest 35S rDNA site was located on the short arm of a submetacentric pair, adjacent to a 5S rDNA site (red arrows in Figure 2c and lower row in Figure. 2d). The long arm of this chromosome exhibited two other 5S rDNA and the largest DAPI ${ }^{+}$band of the complement. Observe that the centromere in the metacentric pair was $\mathrm{DAPI}^{+} / \mathrm{CMA}^{-}$whereas in the other pair it was negative for DAPI and undifferentiated for CMA. The 5S rDNA sites on both chromosome pairs were positively differentiated by CMA.

In $C$. indecora, the number of CMA and DAPI bands was smaller than in C. monogyna. Large $\mathrm{DAPI}^{+}$bands were only observed in the terminal region of a single chromosome pair and in the proximal region of another pair (red arrows in Figure 3a and insets in Figure 3b). Additionally, there was a single proximal band in most chromosomes and several weakly differentiated interstitial and terminal $\mathrm{DAPI}^{+}$bands (Figure 3a). The largest $\mathrm{CMA}^{+}$band was located on the proximal region of a metacentric pair (upper insets in Figure 3b) and several fine interstitial or terminal $\mathrm{CMA}^{+}$bands were observed (Figure 3b). Early-pachytene cells showed a much smaller number of heterochromatic bands in C. indecora when compared with C. monogyna, with a predominance of DAPI ${ }^{+}$bands (Figure $3 \mathrm{~d}, \mathrm{e}$ ).

Concerning rDNA sites, $C$. indecora exhibited five pairs of 5S rDNA sites, all of which were colocalized with $\mathrm{CMA}^{+}$bands, which were sometimes poorly differentiated (Figure 3b, c, and 3f, g). The largest 5S rDNA site was located on the long arm termini of the only submetacentric pair, co-localized with a $\mathrm{CMA}^{+}$band (green arrows in Figure $3 \mathrm{a}, \mathrm{b})$. There were only two pairs of proximal $35 \mathrm{~S}$ rDNA sites (Figure 3c), the largest of which was colocalized with the largest $\mathrm{CMA}^{+}$band (Figure 3b, c), and sometimes distended as a secondary constriction. One or two pairs of small 35S rDNA sites were sometimes observed (white arrows in Figure $3 \mathrm{~g}$ ). Although all rDNA sites appear to colocalize with CMA bands, some $\mathrm{CMA}^{+}$ bands did not colocalized with none of the rDNA sites (compare Figure. 3b, c, and 3 Figure).

After FISH, DAPI stained chromosomes of both species revealed numerous small bands and a few relatively large ones (Figure 4a, b). Most of these bands corresponded to the DAPI bands observed in the direct CMA/DAPI staining, which were now best contrasted. In C. monogyna they were observed as very fine interstitial dot-like bands and a few proximal larger ones (Figure $4 \mathrm{a}$ ), whereas in $C$. indecora there were proximal bands in most chromosomes, a few terminal ones and several weak interstitial bands (Figure 4b). The number of DAPI-FISH bands per chromosome arm varied from 0 to 6 in C. monogyna and from 0 to 5 in $C$. indecora. 


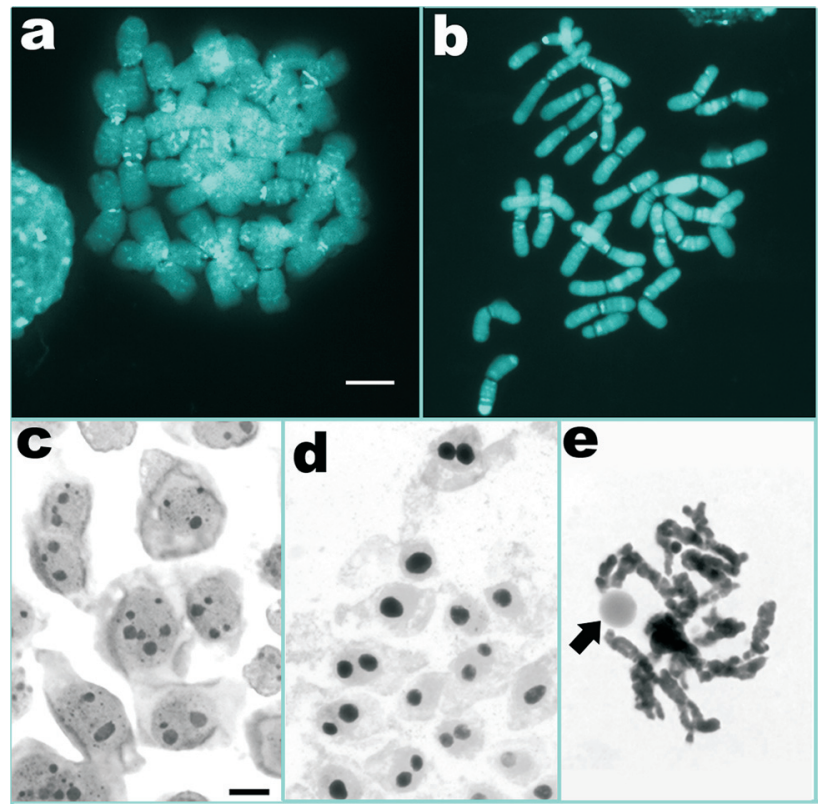

Figure 4 - Heterochromatic bands observed after FISH (a, b) and nucleoli (c-e) in C. monogyna (a, c) and C. indecora (b, d, e). Arrow in (e) points to the single nucleolus of a diplotene. Bar in (a) and (c) corresponds to 10 $\mu \mathrm{m}$.

In order to evaluate if the proliferation of $35 \mathrm{~S} \mathrm{rDNA}$ sites resulted in a proportionally large number of nucleoli, we analyzed 3,052 nuclei of C. monogyna and 1,225 nuclei of $C$. indecora by silver staining. In C. monogyna, the number of nucleoli varied from one to six, with most nuclei displaying two large nucleoli and a few smaller ones (Figure 4c). The number of nucleoli per nucleus in most cells of $C$. indecora was only one or two (Figure 4d), while a very few cells had three or four nucleoli. In all pachytene cells of $C$. indecora only one nucleolus was found (Figure 4e).

\section{Discussion}

\section{Genome size variation}

Our results confirmed that both species possess large DNA content, although the genome size estimated for $C$. indecora $(2 \mathrm{C}=45.58$ and $50.03 \mathrm{pg})$ was much lower than that reported by McNeal et al. (2007) $(2 \mathrm{C}=65.54 \mathrm{pg})$. The different $2 \mathrm{C}$ values observed in the three measured samples for $C$. indecora is too high to be attributed to intraspecific variation (Greilhuber, 2005). The chromosome number of the sample quantified by McNeal et al. (2007) was not determined, but all other previous chromosome counts for $C$. indecora (Fogelberg, 1938; Raven et al., 1965; Pinkava et $a l ., 1974)$, including the present three samples exhibited $2 n$ $=30$. The mitotic chromosome size of our sample (between 10.4 to $18.7 \mu \mathrm{m}$ ) was similar to that described by Fogelberg (1938), who found the largest chromosomes had 16-17 $\mu \mathrm{m}$. Morphologically, C. indecora is a variable species, with three varieties, sharing several important floral characters with other closely related species (Costea et al., 2006). Albeit limited, the available molecular evidence (García et al., 2014; Stefanović et al., 2007) suggests that this species is not monophyletic as currently circumscribed (Figure 1). It contains at least two distinct segregates, one of which is more closely related to members of $C$. coryli Engelm. than to other individuals of $C$. indecora. This phylogenetic distinction among populations of $C$. indecora is consistent with the diversity of genome size measurements reported here and previously (McNeal et al., 2007). Cuscuta coryli, one of the two other species of section Indecorae, has $2 \mathrm{n}=$ 30 medium sized (4 to $8 \mu \mathrm{m}$ ) chromosomes (Fogelberg, 1938), suggesting a $2 \mathrm{C}$ value much smaller than in $C$. indecora and an intense genome size variation inside the section.

The genome size of C. monogyna $(2 \mathrm{C}=67.58 \mathrm{pg})$ is the largest one registered for Cuscuta species. The large genome size of $C$. monogyna and $C$. indecora is mirrored by the large size of their pollen grains; the former species exhibits the largest pollen grains in the genus (Welsh et al., 2010). Actually, C. monogyna has one of the largest genomes reported for eudicots, being surpassed only by some species of Viscaceae and Loranthaceae (Leitch and Leitch, 2013). The $2 \mathrm{C}$ values of 1.7 to $2.4 \mathrm{pg}$ (Ozias-Akins and Jarret, 1994; Bennett and Leitch, 2011) reported for other diploid species of Convolvulaceae with $2 n=30$ or nearly 30 , are at least 17 times lower than those of $C$. monogyna, leading to the hypothesis that large bursts of genome expansion occurred only in the genus Cuscuta. Although the exact phylogenetic position of Cuscuta within Convolvulaceae is still unknown (Stefanović and Olmstead, 2004), Monogynella shares some plesiomorphic features with nonparasitic Convolvulaceae relatives, such as the presence of xylem absent in the remaining subgenera, and some floral, fruit, and anatomical characters (García et al., 2014; Wright et al., 2011).

Unlike those from the subgenus Monogynella, the genome sizes known for the subgenus Grammica are at least three times smaller than that of C. indecora (McNeal et al., 2007). Similar up-and-down variation of genome size has been observed in some other plant taxa (Vallès et al., 2013; Pellicer et al., 2018), but rarely on such a large scale and at such a low phylogenetic level, within a relatively small genus. A similar example is found in the genus Oxalis (Oxalidaceae), with two peaks of high $2 \mathrm{C}$ values: one in the subgenus Oxalis (range: 0.58 to $14.59 \mathrm{pg}$ ) and another in the subgenus Thamnoxys (range:1.76 to $41.88 \mathrm{pg}$ ), with a 72 -fold total variation (Vaio et al., 2018). It is also noteworthy that the two largest genome expansions observed in Cuscuta species resulted in almost identical symmetric karyotypes, while in Oxalis they were quite distinct and asymmetrical. 


\section{Karyotype symmetry}

Assuming that the large genome expansion events were mainly due to amplification of mobile elements (El Baidouri and Panaud, 2013; Garrido-Ramos, 2017), the karyotype symmetry would: a) increase, if insertions of the new elements were equally distributed in the chromosome arms; b) decrease, if the new insertions were preferentially accumulated in some chromosome arms (Levin, 2002; Peruzzi et al., 2009). Given that all Cuscuta subg. Grammica species cytologically known (García and Castroviejo, 2003; García et al., 2019), as well as the non-Cuscuta Convolvulaceae species (Pitrez et al., 2008 and references therein), display small chromosomes and symmetrical to moderately symmetrical karyotypes, we conclude that the two genomes expansions in Cuscuta occurred mainly by proliferation of repetitive elements which were distributed evenly along the length of the chromosome arms.

\section{Heterochromatin and rDNA sites}

Repetitive DNA families represent over $70 \%$ of plant genomes (Michael, 2014), but for these two Cuscuta species, the high number of heterochromatic bands and rDNA sites have contributed greatly to the increasing of these genomes, in comparison to the other species of this group. The elevated number of $5 \mathrm{~S}$ and $35 \mathrm{~S}$ rDNA sites observed in C. indecora (14 sites) and C. monogyna (ca. 66 sites) seems to confirm the correlation between genome size and number of rDNA sites (Prokopowich et al., 2003; Vallès et al., 2013). However, C. nevadensis, with $2 \mathrm{n}=30$ and much smaller chromosomes, had 16 rDNA sites (Ibiapino et al., 2019); therefore, this relationship is not clear for Cuscuta species.

Several $\mathrm{CMA}^{+}$bands were colocalized with rDNA sites but the number of $\mathrm{CMA}^{+}$bands in both Cuscuta species was higher than the number of rDNA sites, indicating that this kind of heterochromatin should be composed by at least three different types of repetitive sequences (5S rDNA, 35S rDNA, and at least a GC-rich satellite DNA sequence corresponding to the $\mathrm{CMA}^{+}$bands which did not colocalize with rDNA sites). In general, $35 \mathrm{~S}$ rDNA sites are positively stained with CMA due to the high GC content of their internal transcribed spacers (ITS) (Baldwin et al., 1995) whereas the non-transcribed spacers (NTS) of $5 \mathrm{~S}$ rDNA sites are more variable in GC content (Waminal et al., 2014), and less often $\mathrm{CMA}^{+}$(e.g., Cabral et al., 2006). In C. indecora and C. monogyna not all 5S and 35S rDNA sites were clearly differentiated with CMA, either because the sites were too small or because they presented a variable GC content. In three other species of Cuscuta subgenus Grammica investigated with sequential CMA/DAPI and FISH staining (Ibiapino et al., 2019), only the 35S rDNA sites were $\mathrm{CMA}^{+}$, indicating a less variable composition of their rDNA repeats.

After the FISH procedure, all DAPI ${ }^{+}$bands observed by CMA/DAPI staining became better contrasted and some other bands not detected before became visible, mainly in $C$. indecora, indicating that part of the heterochromatin was neither particularly rich in $\mathrm{GC}\left(\mathrm{CMA}^{+}\right.$bands) nor in $\mathrm{AT}$ (DAPI ${ }^{+}$bands) (Barros e Silva and Guerra, 2010). Altogether, the number of heterochromatic bands in C. monogyna and $C$. indecora seemed to represent a significant fraction of these large genomes. However, it was not possible to estimate the proportion of heterochromatin in these karyotypes, because most bands were too small and poorly contrasted to allow a reliable measurement. Recent analyses of the plant "satellitome" by NGS and FISH, revealed a surprising diversity of satellite DNA sites (González et al., 2017; Wang et al., 2017; Robledillo et al., 2018), suggesting that the total amount of heterochromatin in these two species may be still higher than observed by banding methods.

In spite of the much higher number of $35 \mathrm{~S}$ rDNA sites in C. monogyna than in C. indecora, the expression of these sites, as estimated by the maximum number of nucleoli per nucleus, was relatively small and similar in both species, possibly because some of them were permanently inactivated, as in Arabidopsis thaliana (Chandrasekhara et al., 2016), or temporarily inactive, as observed in other species (Grabiele et al., 2018; Báez et al., 2020). Thus, the exceeding number of rDNA sites is most likely an accidental consequence of the genome expansion rather than a selective advantage fixed during the evolution of these species.

Beside the difference in number of heterochromatic bands and rDNA sites, C. monogyna and C. indecora presented different distribution patterns of these markers. They were predominantly located on the proximal or terminal chromosome regions in C. indecora and randomly distributed in C. monogyna, suggesting that different mechanisms of rDNA site dispersion were involved. An equilocal distribution of tandem repeats, either terminal or proximal, could be promoted by non-homologous recombination between telomeric or pericentromeric regions of different chromosome pairs (Schweizer and Loidl, 1987; Pedrosa-Harand et al., 2006) 2006) during the bouquet formation or Rabl orientation, an aleatory distribution of repeat arrays is most probably mediated by mobile elements (Dubcovsky and Dvorák, 1995; Raskina et al., 2008; Bueno et al., 2016).

\section{Conclusions}

The huge genome expansion that occurred in two independent Cuscuta lineages included intensive amplification of tandemly repeated sequences without important changes in the karyotype symmetry. Our results indicate that the tandem repeats did not generate large blocks of heterochromatin but rather dozens of small heterochromatic blocks. However, the dispersed fine blocks were not enough to change the original karyotype symmetry of these species. Despite the exceptionally high number of rDNA sites, the maximum number of nucleoli per nucleus observed was relatively low, suggesting that many of these sites were permanently or temporarily inactivated. Further 
analyses of methylation pattern and more specific transcription experiments are necessary to demonstrate the functionality and the faith of these sites.

\section{Acknowledgments}

We are grateful to Dr Sandra Mendes for her valuable technical help. This research was supported by Conselho Nacional de Desenvolvimento Científico e Tecnológico (CNPq), Brazil (grant numbers 308903/2011-0, 311924/2016-6 to M. Guerra), and NSERC of Canada Discovery [grants to M. Costea (327013) and S. Stefanović (326439)].

\section{Conflict of Interest}

The authors declare that there is no conflict of interest related to this study.

\section{Author Contributions}

MAG, MG conceived the study; MAG, MC, SS collected and identified plant material; AI, MG conducted the cytogenetic experiments and wrote the manuscript draft; MAG, MC, SS reviewed the manuscript draft and added several suggestions; all authors read and approved the final version.

\section{References}

Báez M, Souza LGR and Guerra M (2020) Does the chromosomal position of $35 \mathrm{~S}$ rDNA sites influence their transcription? A survey on Nothoscordum species (Amaryllidaceae). Genet Mol Biol 43:e20180194.

Baldwin BG, Sanderson MJ, Porter JM, Wojciechowski MF, Campbell CS and Donoghue MJ (1995) The ITS region of nuclear ribosomal DNA: a valuable source of evidence on angiosperm phylogeny. Ann Mo Bot Gard 82:247-277.

Barros e Silva A and Guerra M (2010) The meaning of DAPI bands observed after C-banding and FISH procedures. Biotech Histochem 85:115-125.

Bennett MD and Leitch IJ (2011) Nuclear DNA amounts in angiosperms: targets, trends and tomorrow. Ann Bot 107:467590.

Bueno D, Palacios-Gimenez OM, Martí DA, Mariguela TC and Cabral-de-Mello DC (2016) The 5S rDNA in two Abracris grasshoppers (Ommatolampidinae: Acrididae): molecular and chromosomal organization. Mol Genet Genomics 291:1607-1613.

Cabral JS, Felix LP and Guerra M (2006) Heterochromatin diversity and its co-localization with $5 \mathrm{~S}$ and $45 \mathrm{~S}$ rDNA sites in chromosomes of four Maxillaria species (Orchidaceae). Genet Mol Biol 29:659-664.

Chandrasekhara C, Mohannath G, Blevins T, Pontvianne F and Pikaard CS (2016) Chromosome-specific NOR inactivation explains selective rRNA gene silencing and dosage control in Arabidopsis. Gene Dev 30:177-190.

Costea M, Nesom GL and Stefanović S (2006) Taxonomy of the Cuscuta indecora (Convolvulaceae) complex in North America. Sida 22:209-225.
Costea M, García MA and Stefanović S (2015) A phylogenetically based infrageneric classification of the parasitic plant genus Cuscuta (Dodders, Convolvulaceae). Syst Bot 40:269-285.

Doleel J (1991) Flow cytometric analysis of nuclear DNA content in higher plants. Phytochem Anal 2:143-154.

Dubcovsky J and Dvorák J (1995) Ribosomal RNA multigene loci: nomads of the Triticeae genomes. Genetics 140:13671377.

El Baidouri M and Panaud O (2013) Comparative genomic paleontology across plant kingdom reveals the dynamics of TE-driven genome evolution. Genome Biol Evol 5:954-965.

Fogelberg SO (1938) The Cytology of Cuscuta. J Torrey Bot Soc 65:631-645.

García MÁ and Castroviejo S (2003) Estudios citotaxonómicos en las especies ibéricas del género Cuscuta (Convolvulaceae). An Jard Bot Madrid 60:33-44.

García MÁ, Costea M, Kuzmina M and Stefanović S (2014) Phylogeny, character evolution, and biogeography of Cuscuta (dodders; Convolvulaceae) inferred from coding plastid and nuclear sequences. Am J Bot 101:670-690.

García MÁ, Costea M, Guerra M, García-Ruiz I and Stefanović S (2019) IAPT chromosome data 31/8. In: Marhold K. and Kučera J. (eds.), IAPT chromosome data 31 - Extended version. Taxon 68:E1-E44.

Garrido-Ramos M (2017) Satellite DNA: An evolving topic. Genes 8:230.

González ML, Chiapella JO and Urdampilleta JD (2017) Characterization of some satellite DNA families in Deschampsia antarctica (Poaceae). Polar Biol 41:457-468.

Grabiele M, Debat HJ, Scaldaferro MA, Aguilera PM, Moscone EA, Seijo JG and Ducasse DA (2018) Highly GC-rich heterochromatin in chili peppers (Capsicum-Solanaceae): A cytogenetic and molecular characterization. Sci Hortic 238:391399.

Greilhuber J, Borsch T, Müller K, Worberg A, Porembski S and Barthlott W (2006) Smallest angiosperm genomes found in Lentibulariaceae, with chromosomes of bacterial size. Plant Biol 8:770-777.

Guerra M (1986) Reviewing the chromosome nomenclature of Levan et al. Rev Bras Genet 9:741-743.

Guerra M (1993) Cytogenetics of Rutaceae. V. High chromosomal variability in Citrus species revealed by CMA/DAPI staining. Heredity 71:234-241.

Guerra M and García MA (2004) Heterochromatin and rDNA sites distribution in the holocentric chromosomes of Cuscuta approximata Bab. (Convolvulaceae). Genome 47:134-140.

Guerra M, Ribeiro T and Felix LP (2019) Monocentric chromosomes in Juncus (Juncaceae) and implications for the chromosome evolution of the family. Bot $J$ Linn Soc 191:475-483.

Ibiapino A, García MA, Ferraz ME, Costea M, Stefanović S and Guerra M (2019) Allopolyploid origin and genome differentiation of the holoparasite species Cuscuta veatchii revealed by genomic in situ hybridization. Genome 62:467-475.

Kaul MLH and Bhan AK (1974) Cytology of Cuscuta reflexa Roxb. Cytologia 39:493-498.

Kodama Y, Yoshida MC and Sasaki M (1980) An improved silver staining technique for nucleolus organizer regions by using nylon cloth. J Hum Genet 25:229. 
Kubeová M, Moravcova L, Suda J, Jaroík V and Pyek P (2010) Naturalized plants have smaller genomes than their noninvading relatives: a flow cytometric analysis of the Czech alien flora. Preslia 82:81-96.

Leitch IJ and Leitch AR (2013) Genome size diversity and evolution in land plants. In: Leitch IJ, Greilhuber J, Doleel J and Wendel JF (eds) Plant Genome Diversity, Volume 2, Physical Structure, Behaviour and Evolution of Plant Genomes. 1nd edition. Springer-Verlag, Wien, pp 307-322.

Leitch IJ, Beaulieu JM, Chase MW, Leitch AR and Fay MF (2010) Genome size dynamics and evolution in monocots. J Bot 2010:1-18.

Levin DA (2002) The Role of Chromosomal Change in Plant Evolution. 1rd edition. Oxford University Press, New York, 240p.

Loureiro J, Rodriguez E, Doleel J and Santos C (2007) Two new nuclear isolation buffers for plant DNA flow cytometry: A test with 37 species. Ann Bot 100:875-888.

McNeal JR, Arumugunathan K, Kuehl JV, Boore JL and dePamphilis CW (2007) Systematics and plastid genome evolution of the cryptically photosynthetic parasitic plant genus $\mathrm{Cus}$ cuta (Convolvulaceae). BMC Biol 5:55.

Michael TP (2014) Plant genome size variation: bloating and purging DNA. Brief Funct Genomics 13:308-317.

Ozias-Akins P and Jarret RL (1994) Nuclear DNA content and ploidy levels in the genus Ipomoea. J Am Soc Hortic Sci 119:110-115.

Palacios-Gimenez OM, Dias GB, Lima LG, Ramos É, Martins C and Cabral-de-Mello DC (2017) High-throughput analysis of the satellitome revealed enormous diversity of satellite DNAs in the neo-Y chromosome of the cricket Eneoptera surinamensis. Sci Rep 7:6422.

Pazy B and Plitmann U (1995) Chromosome divergence in the genus Cuscuta and its systematic implications. Caryologia 48:173-180.

Pedrosa A, Sandal N, Stougaard J, Schweizer D and Bachmair A (2002) Chromosomal map of the model legume Lotus japonicus. Genetics 161:1661-1672.

Pedrosa-Harand A, de Almeida CCS, Mosiolek M, Blair MW, Schweizer D and Guerra M (2006) Extensive ribosomal DNA amplification during Andean common bean (Phaseolus vulgaris L.) evolution. Theor Appl Genet 112:924-933.

Pellicer J, Hidalgo O, Dodsworth S and Leitch IJ (2018) Genome size diversity and its impact on the evolution of land plants. Genes 9:88.

Peruzzi L, Leitch IJ and Caparelli KF (2009) Chromosome diversity and evolution in Liliaceae. Ann Bot 103:459-475.

Pinkava DJ, Brown RK, Lindsay JH and McGill LA (1974) IOPB chromosome numbers reports, XLIV. Taxon 23:379.

Pitrez SR, de Andrade LA, Alves LIF and Felix LP (2008) Karyology of some Convolvulaceae species occurring in NE Brazil inselbergs. Plant Syst Evol 276:235-241.

Prokopowich CD, Gregory TR and Crease TJ (2003) The correlation between rDNA copy number and genome size in eukaryotes. Genome 46:48-50.

Raskina O, Barber JC, Nevo E and Belyayev A (2008) Repetitive DNA and chromosomal rearrangements: speciation-related events in plant genomes. Cytogenet Genome Res 120:351357.
Raven P, Kyhos D and Hill A (1965) Chromosome numbers of spermatophytes, mostly Californian. Aliso 6:105-113.

Robledillo LÁ, Koblíková A, Novák P, Böttinger K, Vrbová I, Neumann P, Schubert I and Macas J (2018) Satellite DNA in Vicia faba is characterized by remarkable diversity in its sequence composition, association with centromeres, and replication timing. Sci Rep 8:5838.

Ruiz-Ruano FJ, López-León MD, Cabrero J and Camacho JPM (2016) High-throughput analysis of the satellitome illuminates satellite DNA evolution. Sci Rep 8:28333.

Schweizer D and Loidl J (1987) A model for heterochromatin dispersion and the evolution of C-band patterns. In: Stahl A, Luciani JM and Vagner-Capodano AM (eds) Chromosomes Today. Springer, Dordrecht, pp 61-74.

Stefanović S and Costea M (2008) Reticulate evolution in the parasitic genus Cuscuta (Convolvulaceae): Over and over again. Botany 86:791-808.

Stefanović S and Olmstead RG (2004) Testing the phylogenetic position of a parasitic plant (Cuscuta, Convolvulaceae, Asteridae): Bayesian inference and the parametric bootstrap on data drawn from three genomes. Syst Biol 53:384-399.

Stefanović S, Krueger L and Olmstead RG (2002) Monophyly of the Convolvulaceae and circumscription of their major lineages based on DNA sequences of multiple chloroplast loci. Am J Bot 89:1510-1522.

Vaio M, Nascimento J, Mendes S, Ibiapino A, Felix LP, Gardner A, Emshwiller E, Fiaschi P and Guerra M (2018) Multiple karyotype changes distinguish two closely related species of Oxalis (O. psoraleoides and O. rhombeo-ovata) and suggest an artificial grouping of section Polymorphae (Oxalidaceae). Bot J Linn Soc 188:269-280.

Vallès J, Canela MÁ, Garcia S, Hidalgo O, Pellicer J, SánchezJiménez I, Siljak-Yakovlev S, Vitales D and Garnatje T (2013) Genome size variation and evolution in the family Asteraceae. Caryologia 66:221-235.

Waminal NE, Ryu KB, Park BR and Kim HH (2014) Phylogeny of Cucurbitaceae species in Korea based on 5S rDNA nontranscribed spacer. Genes Genomics 36:57-64.

Wang G, He Q, Macas J, Novák P, Neumann P, Meng D, Zhao H, Guo N, Han S, Zong M et al. (2017) Karyotypes and distribution of tandem repeat sequences in Brassica nigra determined by fluorescence in situ hybridization. Cytogenet Genome Res 152:158-165.

Welsh M, Stefanović S and Costea M (2010) Pollen evolution and its taxonomic significance in Cuscuta (dodders, Convolvulaceae). Plant Syst Evol 285:83-101.

Wright MA, Welsh M and Costea M (2011) Diversity and evolution of the gynoecium in Cuscuta (dodders, Convolvulaceae) in relation to their reproductive biology: two styles are better than one. Plant Syst Evol 296:51-76.

Yen DE, Gaffey PM and Coates DJ (1992) Chromosome numbers of Australian species of Ipomoea L. (Convolvulaceae). Austrobaileya 3:749-755.

Yuncker TG (1932) The genus Cuscuta. Mem Torrey Bot Club 18:109-331.

Associate Editor: Guilherme Corrêa de Oliveira

License information: This is an open-access article distributed under the terms of the Creative Commons Attribution License (type CC-BY), which permits unrestricted use, distribution and reproduction in any medium, provided the original article is properly cited. 